\title{
Consenso de Especialistas sobre o Implante por Cateter de Biopróteses Valvares para o Tratamento da Estenose Aórtica de Alto Risco Cirúrgico: Relato da Sociedade Brasileira de Hemodinâmica e Cardiologia Intervencionista
}

\author{
Força de Trabalho de Especialistas em Terapia Valvar Aórtica da SBHCl: \\ Fábio Sândoli de Brito Júnior ${ }^{1}$, Pedro Alves Lemos $\mathrm{Neto}^{2}$, Dimytri Siqueira ${ }^{3}$, Rogério Sarmento-Leite ${ }^{4}$, \\ José Armando Mangione ${ }^{5}$, Luiz Antonio Carvalho ${ }^{6}$, Alexandre Abizaid ${ }^{3}$, Deborah C. Nercolini' \\ Marco A. Perin ${ }^{1}$, Marcelo Queiroga ${ }^{8}$, Maurício de Rezende Barbosa9 ${ }^{9}$, \\ Eberhard Grube ${ }^{10}$, J. Eduardo Sousa ${ }^{3,11}$
}

\section{RESUMO}

A substituição cirúrgica da valva aórtica é o tratamento de eleição para pacientes com estenose aórtica sintomática acentuada. Entretanto, o risco cirúrgico aumenta expressivamente com o avançar da idade e com a associação de comorbidades. Nessa situação clínica, o implante por cateter de bioprótese valvar aórtica deve ser considerado uma alternativa terapêutica válida e eficaz. O propósito deste Consenso é oferecer uma revisão crítica do conjunto de evidências e dos avanços mais recentes relacionados ao implante por cateter de bioprótese valvar aórtica e sumarizar o estado da arte atual, com o intuito de melhor guiar a tomada de decisão terapêutica. A seleção dos pacientes para o implante por cateter de prótese valvar aórtica deve obedecer aos seguintes critérios: 1) presença de estenose valvar aórtica acentuada, sintomática, definida como orifício valvar aórtico com área $<1 \mathrm{~cm}^{2}\left(\right.$ ou $\left.<0,6 \mathrm{~cm}^{2} / \mathrm{m}^{2}\right)$; 2) idade $>75$ anos e alta probabilidade de morbidade e mortalidade cirúrgicas pela presença de comorbidades; e

\author{
ABSTRACT \\ Expert Consensus on Transcatheter Aortic-Valve \\ Implantation for the Treatment of High Surgical \\ Risk Aortic Stenosis: Report of the \\ Brazilian Society of Hemodynamics and \\ Interventional Cardiology
}

Surgical replacement of the aortic valve is the therapy of choice for patients with severe symptomatic aortic stenosis. However, surgical risk increases significantly with age and with the association of comorbidities. In this clinical context, transcatheter aortic-valve implantation must be considered as a valid and effective therapeutic alternative. The purpose of this Consensus is to provide a critical review of all the evidence and recent advances related to transcatheter aorticvalve implantation and summarize the current state of the art, in order to better guide therapeutic decision-making. Patient selection for transcatheter aortic-valve implantation must meet the following criteria: 1) presence of severe symp-

\footnotetext{
Hospital Albert Einstein - São Paulo, SP, Brasil.

Hospital Sírio Libanês - São Paulo, SP, Brasil.

3 Instituto Dante Pazzanese de Cardiologia - São Paulo, SP, Brasil.

${ }^{4}$ Instituto de Cardiologia do Rio Grande do Sul - Fundação Universitária de Cardiologia (IC-FUC) - Porto Alegre, RS, Brasil.

${ }^{5}$ Hospital Beneficência Portuguesa de São Paulo - São Paulo, SP, Brasil.

${ }^{6}$ Hospital Pró-Cardíaco - Rio de Janeiro, RJ, Brasil.

7 Hospital Universitário Evangélico de Curitiba - Curitiba, PR, Brasil.

${ }^{8}$ Cardiocenter - Hospital Santa Paula - João Pessoa, PB, Brasil.

${ }^{9}$ Hospital Biocor - Belo Horizonte, MG, Brasil.

${ }^{10}$ Hospital Alemão Oswaldo Cruz - São Paulo, SP, Brasil.

11 Hospital do Coração - Associação do Sanatório Sírio - São Paulo, SP, Brasil.

Correspondência: Fábio Sândoli de Brito Júnior. Rua Dom Armando Lombardi, 819/82-A - São Paulo, SP, Brasil - CEP 05616-011

E-mail: fsbrito@einstein.br

Recebido em: 22/6/2011 • Aceito em: 23/6/2011
} 
3) presença de condição anatômica e morfológica favorável para o procedimento por cateter. As evidências científicas atuais indicam que esse procedimento melhora os sintomas e reduz a mortalidade de pacientes com estenose aórtica e com contraindicação cirúrgica, quando comparado ao tratamento conservador. Adicionalmente, em pacientes com estenose aórtica acentuada e alto risco cirúrgico, o implante por cateter apresenta-se como alternativa terapêutica eficaz, capaz de alterar o curso natural da doença de forma equivalente ao tratamento cirúrgico convencional.

DESCRITORES: Estenose da valva aórtica. Implante de prótese de valva cardíaca. Bioprótese. Cateteres.
A estenose aórtica é a doença valvar cardíaca mais comum. Sua prevalência aumenta com a idade, afetando aproximadamente $3 \%$ da população com idade superior a 75 anos. ${ }^{1}$ A substituição cirúrgica da valva aórtica é, há décadas, o tratamento de eleição para pacientes com estenose aórtica acentuada, propiciando alívio dos sintomas e aumento da sobrevida. Anualmente, cerca de 200 mil cirurgias para substituição valvar aórtica são realizadas no mundo. ${ }^{2}$ Entretanto, o risco cirúrgico aumenta expressivamente com o avançar da idade e com a associação de comorbidades, o que faz com que mais de um terço dos octogenários com estenose aórtica sintomática seja recusado para a cirurgia. ${ }^{3,4} \mathrm{~A}$ impossibilidade cirúrgica nesses casos constitui frequente e importante problema no dia-a-dia da prática médica, especialmente ao se considerar que o aumento da expectativa de vida da população brasileira deve tornar tal fato progressivamente ainda mais comum.

É nesse contexto de busca de alternativas terapêuticas menos invasivas para pacientes com estenose aórtica de alto risco que se insere o implante por cateter de biopróteses valvares aórticas. A experiência clínica, o aprimoramento técnico e o conhecimento científico sobre o tratamento da valva aórtica por cateter crescem rapidamente, com a ampliação da utilização desse procedimento em todo o mundo. O propósito deste Consenso é, portanto, oferecer uma revisão crítica do conjunto de evidências e dos avanços mais recentes nessa área, e sumarizar o estado da arte atual, com o intuito de melhor guiar a tomada de decisão terapêutica.

\section{RECOMENDAÇÕES DO CONSENSO}

I. A estenose aórtica é doença valvar cardíaca comum, cuja prevalência aumenta com a idade.

II. A substituição cirúrgica da valva aórtica é o tratamento de eleição para pacientes com estenose aórtica acentuada. tomatic aortic valve stenosis, defined as aortic valve orifice area $<1 \mathrm{~cm}^{2}$ (or $\left.\left.<0.6 \mathrm{~cm}^{2} / \mathrm{m}^{2}\right) ; 2\right)$ age $>75$ years and a high probability of surgical morbidity and mortality due to pre-existing comorbidities; and 3) the presence of anatomical and morphological conditions favorable for transcatheter interventions. Current scientific evidence indicates that this procedure improves symptoms and reduces mortality in patients with aortic stenosis who were not suitable candidates for surgery, as compared to standard therapy. Additionally, in patients with severe aortic stenosis and high surgical risk, transcatheter aortic-valve implantation is an alternative effective treatment, able to alter the natural course of disease in a manner equivalent to conventional surgical treatment.

KEY-WORDS: Aortic valve stenosis. Heart valve prosthesis implantation. Bioprosthesis. Catheters.

III. No entanto, a contraindicação à cirurgia cardíaca é relativamente comum em idosos com estenose aórtica, em geral relacionada à idade e à presença de comorbidades.

IV. Atualmente, o implante por cateter de bioprótese valvar aórtica deve ser considerado uma alternativa terapêutica válida e eficaz somente para um seleto grupo de pacientes, que, pela idade avançada ou por comorbidades, têm contraindicação ou risco elevado para o tratamento cirúrgico convencional.

V. O tratamento transcateter não pode ser visto como um procedimento de salvamento, não sendo indicado para pacientes em estado clínico ou hemodinâmico terminal.

VI. As evidências atuais indicam, de forma consistente e inequívoca, que o implante por cateter de prótese valvar aórtica é um procedimento seguro e eficaz para abolir a obstrução valvar, determinando queda significativa ou resolução do gradiente de pressão transvalvar aórtico.

VII. As evidências científicas atuais indicam que o implante por cateter de prótese valvar aórtica melhora a qualidade de vida e reduz os sintomas e a mortalidade de pacientes com estenose aórtica e com contraindicação cirúrgica, quando comparado ao tratamento conservador, que pode incluir a valvoplastia aórtica por balão.

VIII. Em pacientes com estenose aórtica acentuada e alto risco cirúrgico, o implante por cateter apresenta-se como alternativa terapêutica eficaz, capaz de alterar o curso natural da doença de forma equivalente ao tratamento cirúrgico convencional.

IX. O respeito bioético à autonomia rege que se deva informar adequadamente o paciente e seus familiares acerca das modalidades terapêuticas (ou seja, tratamento cirúrgico clássico, tratamento por cateter ou manutenção de terapia medicamentosa) e suas implicações prognósticas nos quadros de estenose aórtica grave. 
X. A contraindicação à cirurgia convencional ou a definição do alto risco cirúrgico e, consequentemente, a indicação do tratamento por cateter devem ser, idealmente, conduzidas por equipe médica multidisciplinar.

\section{EVOLUÇÃO DO TRATAMENTO POR CATETER DA ESTENOSE VALVAR AÓRTICA: HISTÓRICO NO BRASIL E NO MUNDO}

Nas últimas décadas, o tratamento por cateter de pacientes com estenose valvar aórtica degenerativa calcificada limitava-se à valvoplastia aórtica com cateter-balão. No entanto, esse procedimento determina melhora apenas temporária dos sintomas e do gradiente de pressão transvalvar, pela alta incidência de reestenose. Tem, portanto, papel terapêutico restrito, sendo atualmente indicado apenas como medida paliativa ou como ponte para o tratamento cirúrgico. 5,6

Em 2002, Cribier et al. ${ }^{7}$ realizaram, com sucesso, o primeiro implante por cateter, em seres humanos, de uma bioprótese valvar aórtica, composta de três folhetos de pericárdio bovino montados em um stent de aço inoxidável expansível por balão, dando início a uma nova era da cardiologia intervencionista. Em 2004, Grube et al. ${ }^{8}$ implantaram, pela primeira vez, uma prótese valvar aórtica autoexpansível. Atualmente, a experiência acumulada com o emprego dessas biopróteses em pacientes com contraindicação ou com alto risco cirúrgico indica que a técnica é segura e eficaz. ${ }^{9-17}$ Até o momento, mais de 30 mil pacientes já foram tratados com esses dispositivos, principalmente em centros europeus, utilizando-se os dois sistemas disponíveis no mundo hoje: a prótese expansível por balão Edwards-SAPIEN ${ }^{\mathrm{TM}}$ (Edwards Lifesciences, Irvine, Estados Unidos) e a prótese autoexpansível CoreValve ${ }^{T M}$ (Medtronic Inc., Minneapolis, Estados Unidos).

A experiência com o implante transcateter de valva aórtica no Brasil iniciou-se em janeiro de 2008, ${ }^{18,19}$ com a aprovação e a disponibilidade do sistema CoreValve ${ }^{\mathrm{TM}}$ para uso regular. Até a data da formulação deste documento, aproximadamente 250 casos já foram realizados em território nacional utilizando esse dispositivo. A prótese Edwards-SAPIEN XT TM, recém-aprovada na Agência Nacional de Vigilância Sanitária (Anvisa), deverá ter o início de sua aplicação em nosso meio em meados de 2011.

Recentemente, com o intuito de monitorar a progressão do uso e os resultados dessa nova modalidade terapêutica, a Sociedade Brasileira de Hemodinâmica e Cardiologia Intervencionista $(\mathrm{SBHCl})$ criou um Registro nacional para acompanhamento dos resultados do implante por cateter de biopróteses aórticas.

\footnotetext{
* Brito Jr. et al. Racional e desenho do Registro Brasileiro de Implante de Bioprótese Aórtica por Cateter. Rev Bras Cardiol Invasiva. 2011. No prelo.
}

\section{INDICAÇÃO DO IMPLANTE TRANSCATETER DE VALVA AÓRTICA: SELEÇÃO DOS PACIENTES}

Atualmente, a indicação do implante por cateter de biopróteses aórticas restringe-se a um seleto grupo de pacientes, que, pela idade avançada ou por comorbidades, têm contraindicação ou elevado risco de morbidade e mortalidade para o tratamento cirúrgico convencional.

A avaliação do risco associado a cirurgias cardíacas constitui-se em processo complexo e comumente subjetivo, representando um desafio a adequada seleção de pacientes para o implante por cateter de bioprótese valvar aórtica.

Diversos índices que combinam dados demográficos, clínicos e variáveis cirúrgicas foram desenvolvidos com o intuito de estimar os riscos associados à cirurgia cardíaca, sendo dois dos mais empregados o EuroSCORE ${ }^{20}$ (www.euroscore.org) e o STS ${ }^{21}$ (www.sts.org/ sections/stsnationaldatabase/riskcalculator). Apesar da correlação somente moderada entre esses índices de risco, ${ }^{22}$ tanto o EuroSCORE como o STS foram amplamente validados em centros de excelência europeus e americanos, sendo largamente utilizados como ferramentas úteis na prática clínica.

É importante notar que índices de avaliação de risco refletem e traduzem o perfil das populações a partir das quais os dados foram extraídos, sendo, por conseguinte, somente aplicáveis a grupos com características semelhantes. Nesse contexto, os variados índices de avaliação de risco não podem ser completamente extrapoláveis ao tipo de paciente com estenose aórtica mencionado anteriormente, uma vez que, tradicionalmente, são recusados para o tratamento cirúrgico e, portanto, sub-representados nas bases de dados utilizadas para derivar esses escores e algoritmos.

Adicionalmente, esses escores de risco podem, por vezes, não traduzir o real risco cirúrgico de um paciente pelo fato de não incluírem diversos fatores que conhecidamente elevam de forma significativa o risco do tratamento cirúrgico. Como exemplo, podese citar a presença de aorta em porcelana, irradiação torácica prévia, hepatopatias e distúrbios da coagulação, ausentes no cálculo do EuroSCORE. Dessa maneira, os escores de risco têm utilidade apenas para apoiar a avaliação clínica individualizada, que persiste soberana.

Comumente, em populações de pacientes geriátricos, aspectos relacionados à "fragilidade" do indivíduo são percebidos como limitantes clínicos a estratégias médicas de maior agressividade. Atualmente, uma avaliação semiquantitativa pode ser obtida por meio de escores de fragilidade, que levam em consideração critérios clínicos, laboratoriais e funcionais. O índice de fragilidade do Columbia University Medical Center/ New York Presbyterian Hospital, utilizado no estudo 
randomizado PARTNER, ${ }^{16,17}$ é um dos escores utilizados em estudos que avaliam o implante transcateter de valva aórtica. É importante notar, no entanto, que as evidências disponíveis no momento não permitem utilizar a avaliação da fragilidade como substituto, mas apenas como ferramenta adicional à avaliação do risco cirúrgico pelos escores validados para esse fim e pela análise clínica.

O alto risco ao tratamento cirúrgico, per se, não constitui indicação para o procedimento por cateter. É parte integrante e essencial da avaliação do candidato ao tratamento a análise de parâmetros anatômicos e morfológicos do complexo aórtico e da via de acesso, objetivando determinar a exequibilidade técnica da substituição valvar por cateter. ${ }^{23}$ Para isso, o ecocardiograma, a angiotomografia de múltiplos detectores, a aortografia e a arteriografia do território ilíaco-femoral podem ser úteis e fornecer as informações essenciais para o planejamento do procedimento. A coronariografia também é recomendada para descartar a presença de doença arterial coronária grave associada.

De maneira geral, a seleção dos pacientes para o implante por cateter de prótese valvar aórtica deve obedecer aos seguintes critérios:

- Presença de estenose valvar aórtica acentuada, sintomática, definida como orifício valvar aórtico com área $<1 \mathrm{~cm}^{2}$ (ou $\left.<0,6 \mathrm{~cm}^{2} / \mathrm{m}^{2}\right)$.

- Idade > 75 anos e alta probabilidade de morbidade e mortalidade cirúrgicas pela presença de comorbidades. São exemplos de tais condições clínicas: insuficiência renal; cirrose hepática; doença pulmonar crônica (por exemplo, FEV1 < $1 \mathrm{~L}$ ou uso de oxigenioterapia domiciliar); múltiplas cirurgias cardíacas prévias, especialmente com enxerto de artéria mamária pérvio; aorta em porcelana; hipertensão arterial pulmonar (> $60 \mathrm{mmHg}$ ); radioterapia torácica prévia; e fragilidade orgânica.

- Presença de condição anatômica e morfológica favorável para o procedimento por cateter, incluindo a avaliação pormenorizada da via de acesso e do trajeto vascular, bem como dos aspectos cardíacos de interesse para a exequibilidade do procedimento. As vias de acesso femoral, subclávia, transaórtica ou transapical têm sido descritas, devendo a escolha da via de acesso ser efetuada por meio de avaliação pormenorizada caso a caso, obedecendo a parâmetros rígidos de seleção, específicos para cada prótese/sistema de entrega. Este Consenso entende que a via de acesso adotada deve ser a menos invasiva possível e que apresente menor possibilidade de complicações. Na prática, observa-se que a via femoral é aquela com maior exequibilidade técnica e menor necessidade de envolvimento de múltiplos profissionais médicos, culminando por ser a mais comumente utilizada no dia-a-dia, o que faz dela, possivelmente, a via de escolha preferencial, quando tecnicamente factível.

\section{IMPLANTE POR CATETER DE BIOPRÓTESES AÓRTICAS: IMPACTO CLÍNICO}

Estudos clínicos recentes têm demonstrado, consistentemente, a exequibilidade, a segurança e a eficácia desse tipo de intervenção, com resultados, ainda que a curto e médio prazos, bastante animadores. ${ }^{9-17,24-27}$ Os achados atuais podem ser resumidos da seguinte forma:

a) O resultado do procedimento está estreitamente relacionado à experiência. Após a superação da curva de aprendizado, o índice de sucesso de cerca de $90 \%$ é sistematicamente relatado, com embolização da prótese e outras complicações relacionadas à técnica ocorrendo mais raramente durante o procedimento. Também, o aumento da experiência dos operadores possibilita o tratamento de pacientes com maior espectro de gravidade, sem elevar ou até minimizando a ocorrência de complicações.

b) Os estudos divulgados são concordantes em demonstrar o adequado funcionamento das biopróteses aórticas implantadas por cateteres. Após o implante, geralmente atinge-se área valvar superior a 1,5 $\mathrm{cm}^{2} \mathrm{e}$ nota-se queda importante ou resolução completa do gradiente de pressão transvalvar aórtico. Essa melhora hemodinâmica precoce se reflete, rapidamente, na melhora dos sintomas de insuficiência cardíaca congestiva dos pacientes tratados, com impacto favorável na qualidade de vida. A médio prazo, frequentemente nota-se melhora da função e redução da hipertrofia do ventrículo esquerdo. Aproximadamente $50 \%$ a $70 \%$ dos casos apresentam regurgitação perivalvar de pequena intensidade ao final da intervenção, que tende a diminuir durante o seguimento. Regurgitação perivalvar acentuada ocorre em torno de $5 \%$ dos casos e pode ser manuseada com redilatações da prótese com balões ou até o implante de prótese adicional. Relatos de degeneração estrutural da prótese na evolução tardia são raríssimos, embora maior tempo de seguimento seja necessário para que tenhamos mais informações sobre o desempenho desses dispositivos a longo prazo.

c) A mortalidade aos 30 dias varia de $5 \%$ a $18 \%$, dependendo da gravidade clínica e da complexidade anatômica dos casos tratados. Deve-se ressaltar, entretanto, que essas taxas de mortalidade são muito inferiores àquelas estimadas pelos escores de risco para o tratamento cirúrgico convencional desses mesmos pacientes. Algumas publicações apresentam seguimento clínico tardio de até 2 anos, com sobrevida de $70 \%$ a $80 \%$ e melhora expressiva da condição clínica da maior parte dos pacientes. ${ }^{15} \mathrm{~A}$ maioria das mortes tardias decorre de comorbidades e não se relaciona a problemas com a prótese.

d) Recentemente, divulgaram-se os dados do estudo randomizado PARTNER, ${ }^{16,17}$ utilizando a bioprótese Edwards-SAPIEN ${ }^{\mathrm{TM}}$. Nesse estudo, a mortalidade após 
um ano de tratamento em pacientes inoperáveis foi reduzida de aproximadamente $50 \%$ com o tratamento clínico convencional para 30\% com o implante por cateter de bioprótese. Nos casos considerados de alto risco cirúrgico, o implante por cateter apresentou mortalidade após um ano equivalente àquela obtida com o tratamento cirúrgico, demonstrando que o implante por cateter é uma excelente opção terapêutica para essa população. Diversos Registros europeus com a bioprótese CoreValve ${ }^{\mathrm{TM}}$ confirmam esses achados. ${ }^{24-27}$

e) Outras complicações relacionadas ao procedimento são descritas nessa população de alto risco e repleta de comorbidades:

- Complicações vasculares no local de acesso ocorrem em $1,9 \%$ a $15 \%$ dos casos e implicam significativa morbidade e mortalidade.

- A incidência clínica de acidente vascular cerebral varia de $1 \%$ a $10 \%$. Essa complicação pode estar relacionada ao ateroembolismo de placas presentes na aorta ascendente e no arco aórtico, à embolização de cálcio e debris dos folhetos da valva aórtica nativa, ao tromboembolismo proveniente da prótese valvar e à eventual ocorrência de fibrilação atrial. Visando à prevenção do acidente vascular cerebral, recomenda-se a prescrição de antiagregação plaquetária dupla sempre que possível por 3 a 6 meses após o implante por cateter das próteses.

- A oclusão de artérias coronárias pelas biopróteses é rara $(<1 \%)$, relacionando-se à presença de calcificação, espessamento ou redundância excessivas dos folhetos aórticos, à altura dos óstios coronários e às dimensões dos seios de Valsalva.

- Dada a estreita relação anatômica do sistema de condução elétrico do coração com o ânulo valvar aórtico e o trajeto dos ramos do feixe de His no septo interventricular, pode-se observar bloqueio completo do ramo esquerdo e diversos graus de bloqueio atrioventricular após o implante por cateter de bioprótese aórtica. Estudos recentes indicam que 20\% a $40 \%$ dos pacientes submetidos a implante da prótese CoreValve $\mathrm{e}^{\mathrm{TM}}$ e 3\% a 5\% com a prótese Edwards-SAPIEN ${ }^{T M}$ têm necessidade de implantar o marca-passo permanente. ${ }^{28-34} \mathrm{~A}$ maior protrusão da CoreValve ${ }^{\mathrm{TM}}$ no trato de saída do ventrículo esquerdo poderia explicar tais diferenças de incidência entre os dispositivos. Ademais, fatores como idade avançada, presença de bloqueio de ramo direito prévio e grau de hipertrofia ventricular podem estar relacionados à maior necessidade de marca-passo permanente. Cabe destacar que esse tipo de problema também pode ocorrer após o tratamento cirúrgico da estenose aórtica. ${ }^{35}$

\section{IMPLICAÇÕES PARA TREINAMENTO E CAPACITAÇÃO DOS INTERVENCIONISTAS}

A aquisição da competência para indicação, seleção dos casos, realização do procedimento e cuidados do paciente submetido a implante por cateter de bioprótese valvar aórtica exibe curva de aprendizado mais prolongada. Idealmente, a equipe envolvida deve ser multidisciplinar, incluindo, além do cardiologista intervencionista, cardiologistas clínicos, cirurgiões cardíacos, anestesiologistas e especialistas em imagem. Os intervencionistas devem possuir extenso conhecimento da anatomia, da fisiopatologia, das alterações hemodinâmicas e da história natural da valvopatia aórtica, bem como dos resultados das várias opções terapêuticas, das possíveis complicações e da evolução tardia após os procedimentos.

O cardiologista intervencionista deve estar preparado para analisar, com o apoio de radiologistas e ecocardiografistas, os exames de angiotomografia e de ecocardiograma, de modo a selecionar, de forma adequada, pacientes que apresentem condições anatômicas favoráveis para o implante por cateter de bioprótese valvar aórtica.

São fundamentais a experiência na cateterização transaórtica do ventrículo esquerdo e na manipulação de múltiplos dispositivos próprios para o procedimento bem como o adequado domínio dos parâmetros hemodinâmicos. Além disso, é necessário preparo para controle de possíveis complicações, como insuficiência aórtica aguda, perfuração cardíaca, tamponamento pericárdico, acidente vascular cerebral, bloqueio atrioventricular e complicações de via de acesso.

Em geral, para se obter proficiência, é necessário realizar um mínimo de 10 procedimentos assistidos por profissionais experientes. Estimula-se a utilização de métodos didáticos auxiliares para ampliar o nível de aprendizado durante as fases de treinamento e manutenção, como o uso de simuladores, prática em modelo animal, e simpósios dedicados ao tema. De modo amplo, a $\mathrm{SBHCl}$ chama a si a responsabilidade de maximizar as possibilidades de acesso ao treinamento e à educação continuada sobre o método terapêutico.

\section{IMPLANTE TRANSCATETER DE VALVA AÓRTICA DIANTE DOS PRINCÍPIOS DA ÉTICA BIOMÉDICA}

A ética biomédica possui princípios básicos que se aplicam ao geral das circunstâncias na prática clínica, provindos de juízos ponderados do interior da moralidade comum e da tradição médica: a autonomia, a não-maleficência, a beneficência e a justiça. No caso particular do implante transcateter de valva aórtica, o enfoque inicial concentra-se no assentimento do paciente para se submeter ao método, sobretudo pelo fato de a maioria dos pacientes alocados para esse procedimento ser constituída por indivíduos com altíssimo risco para realização de cirurgia de troca valvar aórtica a céu aberto ou mesmo com contraindicação a essa técnica. Destaque-se que os pacientes, em grande proporção, são octogenários e nonagenários. 
É nessas circunstâncias que se insere a necessidade da obtenção do consentimento informado, o qual pressupõe que o paciente tenha a capacidade de discernimento e voluntariedade. Embora, habitualmente, se restrinjam informações a idosos, prestando-as quase exclusivamente a seus familiares, estes também têm o direito de participar do processo de consentimento, e é dever do médico fazê-lo. A percepção por parte do médico de que seu paciente, candidato a implante por cateter de valva aórtica, se encontra com autonomia, capacidade de decisão e voluntariedade preservadas deve ser um elemento encorajador no recrutamento desse enfermo para o método. Em outra ponta, naqueles indivíduos idosos, mesmo portadores de estenose valvar aórtica grave, cujas propriedades descritas acima estejam suprimidas, deve ser muito bem sopesada a indicação da terapêutica invasiva.

Como previsto no Código de Ética Médica (CEM) brasileiro, também deve ser considerado se a realização do procedimento contribuirá para proporcionar melhora da qualidade e aumento da sobrevida do paciente. Transcrevemos ipsis verbis conforme consta no Código (parágrafo único do artigo 41): "Nos casos de doença incurável e terminal, deve o médico oferecer todos os cuidados paliativos disponíveis sem empreender ações diagnósticas ou terapêuticas inúteis ou obstinadas, levando sempre em consideração a vontade expressa do paciente ou, na sua impossibilidade, a de seu representante legal." Ainda, em face dos potenciais benefícios do implante por cateter, restringir o acesso dos enfermos graves, sem alternativa terapêutica com a mesma eficácia, poderia ser encarado como ofensa à norma contida no artigo 32 do CEM, o qual veda ao médico: deixar de usar todos os meios disponíveis de diagnóstico e tratamento, cientificamente reconhecidos e a seu alcance, em favor do paciente. Assim, sobretudo naqueles pacientes com contraindicação cirúrgica, o implante por cateter de valva aórtica não pode ser meramente desconsiderado, especialmente à medida que evidências científicas consolidam o método como associado a redução significativa da mortalidade.

No contexto específico do paciente portador de estenose valvar aórtica grave candidato a implante por cateter, em que o cardiologista intervencionista é também o responsável pela condução do caso clínico, deve-se considerar que o contraindicar da cirurgia convencional pode representar um cenário de potencial conflito de interesses. Nessas circunstâncias, é conveniente que sejam documentados no prontuário médico todos os elementos comprobatórios da proscrição do procedimento cirúrgico tradicional. Essa situação é diversa de quando a técnica é indicada para o enfermo por outro especialista ou de quando o paciente já está com impedimento ao tratamento cirúrgico tradicional atestado por um cirurgião cardiovascular, condições tais que envolvem, indiscutivelmente, menor complexidade inerente.

\section{INCORPORAÇÃO DO IMPLANTE POR CATETER DE PRÓTESE VALVAR AÓRTICA À PRÁTICA MÉDICA}

Em virtude dos aspectos apresentados, não resta dúvida sobre a conveniência da adoção do implante transcateter de valva aórtica como medida integrante do rol de políticas públicas de saúde.

Quando analisamos o implante por cateter de bioprótese aórtica, é oportuna a discussão acerca da incorporação de novas técnicas e tecnologias destinadas à atenção à saúde no Brasil:

1. Nas técnicas em que é necessário o emprego de dispositivos, a exemplo de órteses e próteses, como no caso específico do implante por cateter de bioprótese aórtica, é necessário o registro desse produto na Anvisa, que, para sua concessão, dentre outros requisitos, verifica se há condições de segurança e eficácia nas indicações propostas pelo fabricante.

2. Em 2006, o Ministério da Saúde, por meio da Portaria 3.323 do Gabinete do Ministro da Saúde, criou a Comissão de Incorporação de Tecnologias do Ministério da Saúde (CITEC), cujo objetivo é racionalizar e modernizar o fluxo de incorporação de novas tecnologias no Sistema Único de Saúde (SUS) e na Saúde Suplementar. À luz do estabelecido nessa portaria, o implante por cateter de valva aórtica teria que, obrigatoriamente, ser submetido a análise e aprovação da CITEC, a qual avaliaria ou não a conveniência da incorporação dessa técnica/tecnologia no âmbito do SUS e da Saúde Suplementar. A despeito dessas considerações, na prática, a incorporação de novas tecnologias, ao menos no âmbito da Saúde Suplementar, não tem seguido necessariamente esse fluxograma. A Agência Nacional de Saúde, com fulcro na lei 9.656, tem determinado a inclusão dessas novas técnicas mesmo sem a apreciação da CITEC, sobretudo por ter competência legal para tal - no Rol de Procedimentos e Eventos em Saúde. Esse rol é periodicamente atualizado e publicado, após submissão a consulta pública, por meio de resolução normativa própria.

3. Merece ênfase, igualmente, na incorporação de novas tecnologias, o papel exercido pelo Conselho Federal de Medicina, autarquia federal responsável pela fiscalização do exercício profissional, em face de suas atribuições precípuas e também de disposição legal. Nesse particular, da lei 9.656, a qual no inciso IX, do artigo 10, exclui de cobertura pelos planos de saúde os tratamentos ilícitos ou antiéticos, assim definidos sob o aspecto médico ou não reconhecidos pelas autoridades competentes.

4. Por fim, a incorporação de uma nova técnica na política de saúde do Brasil dar-se-á com sua inclusão para reembolso pelos agentes provedores de saúde, públicos ou privados, representados, respectivamente, pelo SUS e pela Agência Nacional de Saúde. 
Como já destacado, apesar dos resultados clínicos favoráveis ao emprego do implante por cateter de bioprótese valvar aórtica, demonstrados na literatura especializada, e de o método já ser praticado na União Europeia e em vários países da América Latina, até o momento essa técnica não foi incluída no Rol de Procedimentos e Eventos em Saúde da Agência Nacional de Saúde, tampouco integra a lista de procedimentos do SUS. A despeito desse cenário adverso, já foram realizados mais de 250 implantes dessa natureza no País.

Tal contexto tem ensejado uma variada série de conflitos judiciais entre pacientes e operadoras de planos de saúde, visando à implementação desse direito. O papel do médico, nesse contexto, assume considerável relevo, pois é com esteio em suas informações, por meio de relatórios circunstanciados, que se produzirá prova para fundamentar a ação judicial. Ao médico não compete fazer juízo de valor acerca da plausibilidade do direito do paciente, mas sim relatar de forma clara e concisa as razões que norteiam sua requisição, em conformidade com o estabelecido nos artigos 86 e 91 do CEM. Nesse intricado panorama, é recomendável ao médico acautelar-se, sempre focado no melhor interesse do paciente.

\section{REFERÊNCIAS}

1. Lindroos M, Kupari M, Heikkila J, Tilvis R. Prevalence of aortic valve abnormalities in the elderly: an echocardiographic study of a random population sample. J Am Coll Cardiol. $1993 ; 21(5): 1220-5$

2. Fann JI, Chronos N, Rowe SJ, Michiels R, Lyons BE, Leon $M B$, et al. Evolving strategies for the treatment of valvular heart disease: preclinical and clinical pathways for percutaneous aortic valve replacement. Catheter Cardiovasc Interv. 2008; 71(3):434-40.

3. Bouma BJ, van Den Brink RB, van Der Meulen $\mathrm{JH}$, Verheul HA, Cheriex EC, Hamer HP, et al. To operate or not on elderly patients with aortic stenosis: the decision and its consequences. Heart. 1999;82(2):143-8.

4. Iung B, Cachier A, Baron G, Messika-Zeitoun D, Delahaye $\mathrm{F}$, Tornos $\mathrm{P}$, et al. Decision-making in elderly patients with severe aortic stenosis: why are so many denied surgery? Eur Heart J. 2005;26(24):2714-20.

5. Kuntz RE, Tosteson AN, Berman AD, Goldman L, Gordon PC, Leonard BM, et al. Predictors of event-free survival after balloon aortic valvuloplasty. N Engl J Med. 1991;325(1):17-23.

6. Otto CM, Mickel MC, Kennedy JW, Alderman EL, Bashore TM, Block PC, et al. Three-year outcome after balloon aortic valvuloplasty: insights into prognosis of valvular aortic stenosis. Circulation. 1994;89(2):642-50.

7. Cribier A, Eltchaninoff $\mathrm{H}$, Bash A, Borenstein N, Tron C, Bauer $F$, et al. Percutaneous transcatheter implantation of an aortic valve prosthesis for calcific aortic stenosis: first human case description. Circulation. 2002;106(24):3006-8.

8. Grube E, Laborde JC, Zickmann B, Gerckens U, Felderhoff T, Sauren B, et al. First report on a human percutaneous transluminal implantation of a self-expanding valve prosthesis for interventional treatment of aortic valve stenosis. Catheter Cardiovasc Interv. 2005;66(4):465-9.

9. Cribier A, Eltchaninoff $\mathrm{H}$, Tron C, Bauer $\mathrm{F}$, Agatiello C, Nercolini D, et al. Treatment of calcific aortic stenosis with the percutaneous heart valve: mid-term follow-up from the initial feasibility studies: the French experience. J Am Coll Cardiol. 2006;47(6):1214-23

10. Cribier A, Eltchaninoff $\mathrm{H}$, Tron C, Bauer F, Agatiello C, Sebagh L, et al. Early experience with percutaneous transcatheter implantation of heart valve prosthesis for the treatment of end-stage inoperable patients with calcific aortic stenosis. J Am Coll Cardiol. 2004;43(4):698-703.

11. Grube E, Laborde JC, Gerckens U, Felderhoff T, Sauren B, Buellesfeld L, et al. Percutaneous implantation of the CoreValve self-expanding valve prosthesis in high-risk patients with aortic valve disease: the Siegburg first-in-man study. Circulation. 2006;114(15):1616-24.

12. Grube E, Schuler G, Buellesfeld L, Gerckens U, Linke A Wenaweser $\mathrm{P}$, et al. Percutaneous aortic valve replacement for severe aortic stenosis in high-risk patients using the second-and current third-generation self-expanding CoreValve prosthesis: device success and 30-day clinical outcome. I Am Coll Cardiol. 2007;50(1):69-76.

13. Piazza N, Grube E, Gerckens U, den Heijer P, Linke A, Luha $\mathrm{O}$, et al. Procedural and 30-day outcomes following transcatheter aortic valve implantation using the third generation $(18 \mathrm{Fr}$ ) CoreValve ReValving System: results from the multicentre, expanded evaluation registry 1 -year following CE mark approval. Eurolntervention. 2008;4(2):242-9.

14. Webb JG, Pasupati S, Humphries K, Thompson C, Altwegg $\mathrm{L}$, Moss R, et al. Percutaneous transarterial aortic valve replacement in selected high-risk patients with aortic stenosis. Circulation. 2007;116(7):755-63.

15. Buellesfeld L, Gerckens U, Schuler G, Bonan R, Kovac J, Serruys PW, et al. 2-year follow-up of patients undergoing transcatheter aortic valve implantation using a self-expanding valve prosthesis. J Am Coll Cardiol. 2011;57(16):1650-7.

16. Leon MB, Smith CR, Mack M, Miller DC, Moses JW, Svensson LG, et al. Transcatheter aortic-valve implantation for aortic stenosis in patients who cannot undergo surgery. N Engl Med. 2010;363(17):1597-607.

17. Smith CR, Leon MB, Mack MJ, Miller DC, Moses JW, Svensson LG, et al. Transcatheter versus surgical aortic-valve replacement in high-risk patients. N Engl J Med. 2011;364(23):2187-98.

18. Leite RS, Quadros AS, Prates PRL, Zanatta LG, Salgado Filho PA, Grando T, et al. Implante valvular aórtico percutâneo: experiência inicial do Sul do Brasil. Rev Bras Cardiol Invasiva. 2008;16(4):398-405.

19. Perin MA, Brito Jr FS, Almeida BO, Pereira MAM, Abizaid A, Tarasoutchi $F$, et al. Substituição valvar aórtica percutânea para o tratamento da estenose aórtica: experiência inicial no Brasil. Arq Bras Cardiol. 2009;93(3):299-306.

20. Roques F, Nashef SA, Michel P; EuroSCORE Study Group. Risk factors for early mortality after valve surgery in Europe in the 1990s: lessons from the EuroSCORE Pilot Program. Heart Valve Dis. 2001;10(5):572-7; discussion 577-8.

21. Hattler BG, Madia C, Johnson C, Armitage JM, Hardesty RL, Kormos RL, et al. Risk stratification using the Society of Thoracic Surgeons Program. Ann Thorac Surg. 1994;58(5): 1348-52.

22. Piazza N, Wenaweser $P$, van Gameren $M$, Pilgrim T, Tzikas A, Otten A, et al. Relationship between the logistic EuroSCORE and the Society of Thoracic Surgeons Predicted Risk of Mortality score in patients implanted with the CoreValve ReValving System-a Bern-Rotterdam Study. Am Heart J. 2010;159(2):323-9.

23. Piazza N, de Jaegere $P$, Schultz C, Becker AE, Serruys PW, Anderson $\mathrm{RH}$. Anatomy of the aortic valvar complex and its implications for transcatheter implantation of the aortic valve. Circ Cardiovasc Intervent. 2008;1(1):74-81. 
24. Bosmans JM, Kefer J, De Bruyne B, Herijgers P, Dubois C, Legrand $V$, et al. Procedural, 30-day and one year outcome following CoreValve or Edwards transcatheter aortic valve implantation: results of the Belgian National Registry. Interact Cardiovasc Thorac Surg. 2011;12(5):762-7.

25. Eltchaninoff $H$, Prat A, Gilard M, Leguerrier A, Blanchard D, Fournial G, et al. Transcatheter aortic valve implantation: early results of the FRANCE (French Aortic National Corevalve and Edwards) registry. Eur Heart J. 2011;32(2):191-7.

26. Tamburino C, Capodanno D, Ramondo A, Petronio AS, Ettori F, Santoro G, et al. Incidence and predictors of early and late mortality after transcatheter aortic valve implantation in 663 patients with severe aortic stenosis. Circulation. 2011; 123(3):299-308.

27. Zahn R, Gerckens U, Grube E, Linke A, Sievert H, Eggebrecht $\mathrm{H}$, et al. Transcatheter aortic valve implantation: first results from a multi-centre real-world registry. Eur Heart J. 2011;32(2): 198-204.

28. Fraccaro C, Buja G, Tarantini G, Gasparetto V, Leoni L, Razzolini R, et al. Incidence, predictors, and outcome of conduction disorders after transcatheter self-expandable aortic valve implantation. Am J Cardiol. 2011;107(5):747-54.

29. Godin M, Eltchaninoff H, Furuta A, Tron C, Anselme F, Bejar $\mathrm{K}$, et al. Frequency of conduction disturbances after transcatheter implantation of an Edwards Sapien aortic valve prosthesis. Am J Cardiol. 2010;106(5):707-12.
30. Khawaja MZ, Rajani R, Cook A, Khavandi A, Moynagh A, Chowdhary $S$, et al. Permanent pacemaker insertion after CoreValve transcatheter aortic valve implantation: incidence and contributing factors (the UK CoreValve Collaborative). Circulation. 2011;123(9):951-60.

31. Roten L, Wenaweser P, Delacretaz E, Hellige G, Stortecky S, Tanner $\mathrm{H}$, et al. Incidence and predictors of atrioventricular conduction impairment after transcatheter aortic valve implantation. Am J Cardiol. 2010;106(10):1473-80.

32. Rubin J, Avanzas P, del Valle R, Morís C. Long-term follow up of atrioventricular block in transcatheter aortic valve implantation. Am J Cardiol. 2011;107(4):641-2.

33. Leite RS, Quadros AS, Prates PRL, Voltolini I, Conti E, Giusti I, et al. Marca-passo permanente após implante percutâneo valvular aórtico: A necessidade é maior que imaginávamos? Rev Bras Cardiol Invasiva. 2009;17(4):476-83.

34. Brito Jr FS, Perin MA, Almeida BO, Abizaid A, Pereira MAM, Caixeta $A$, et al. Incidência de distúrbios da condução atrioventricular e intraventricular após implante percutâneo da bioprótese valvar aórtica CoreValve. Rev Bras Cardiol Invasiva. 2010;18(2):128-34.

35. Dawkins S, Hobson AR, Kalra PR, Tang AT, Monro JL, Dawkins KD. Permanent pacemaker implantation after isolated aortic valve replacement: incidence, indications, and predictors. Ann Thorac Surg. 2008;85(1):108-12.

\section{APÊNDICE \\ Conflito de interesses}

\begin{tabular}{|c|c|c|c|c|c|c|c|}
\hline Nome & $\begin{array}{c}\text { Participação } \\
\text { em estudo }^{1}\end{array}$ & Palestrante $^{2}$ & Consultor $^{3}$ & $\begin{array}{c}\text { Comitê } \\
\text { normativo }^{4}\end{array}$ & $\begin{array}{c}\text { Auxílio pessoal/ } \\
\text { institucional }^{5}\end{array}$ & $\begin{array}{c}\text { Textos } \\
\text { científicos } \\
\text { patrocinados }^{6}\end{array}$ & $\begin{array}{c}\text { Participação } \\
\text { acionária }^{7}\end{array}$ \\
\hline Alexandre Abizaid & Não & Não & Não & Não & Não & Não & Não \\
\hline Deborah C. Nercolini & Não & Sim & Não & Não & Não & Não & Não \\
\hline Dimytri Siqueira & Não & Não & Não & Não & Não & Não & Não \\
\hline Eberhard Grube & Não & Não & Sim & Não & Não & Não & Não \\
\hline Fábio Sândoli de Brito Júnior & Não & Sim & Não & Não & Não & Não & Não \\
\hline J. Eduardo Sousa & Não & Não & Não & Não & Não & Não & Não \\
\hline José Armando Mangione & Não & Sim & Não & Não & Não & Não & Não \\
\hline Luiz Antonio Carvalho & Não & Sim & Não & Não & Não & Não & Não \\
\hline Marcelo Queiroga & Não & Não & Não & Não & Não & Não & Não \\
\hline Marco A. Perin & Não & Sim & Sim & Não & Não & Não & Não \\
\hline Maurício de Rezende Barbosa & Não & Não & Não & Não & Não & Não & Não \\
\hline Pedro Alves Lemos Neto & Não & Não & Não & Não & Não & Não & Não \\
\hline Rogério Sarmento-Leite & Não & Sim & Não & Não & Sim & Não & Não \\
\hline \multicolumn{8}{|c|}{$\begin{array}{l}1 \text { = Participou de estudos clínicos e/ou experimentais subvencionados pela indústria farmacêutica ou de equipamentos relacionados } \\
\text { ao Consenso?; } 2 \text { = Foi palestrante em atividades ou eventos patrocinados pela indústria relacionados ao Consenso?; } 3=\text { Foi (é) } \\
\text { membro de conselho consultivo ou diretivo da indústria farmacêutica ou de equipamentos relacionado ao Consenso?; } 4=\text { Participou } \\
\text { de comitês normativos de estudos científicos patrocinados pela indústria relacionados ao Consenso?; } 5=\text { Recebeu auxílio pessoal ou } \\
\text { institucional da indústria relacionado ao Consenso?; } 6 \text { = Elaborou textos científicos em periódicos patrocinados pela indústria } \\
\text { relacionados ao Consenso?; } 7=\text { Tem ações da indústria relacionadas ao Consenso?. }\end{array}$} \\
\hline
\end{tabular}

